\title{
Palomar testbed interferometer status report
}

Benjamin F. Lane, M. Konacki, Robert Russell Thompson, Gerard Theodore van Belle, M. Mark Colavita, et al.

Benjamin F. Lane, M. Konacki, Robert Russell Thompson, Gerard Theodore van Belle, M. Mark Colavita, Andrew F. Boden, "Palomar testbed interferometer status report," Proc. SPIE 4838, Interferometry for Optical Astronomy II, (21 February 2003); doi: 10.1117/12.456770

SPIE Event: Astronomical Telescopes and Instrumentation, 2002, Waikoloa, Hawai'i, United States 


\title{
Palomar Testbed Interferometer Status Report
}

\author{
B.F. Lane ${ }^{a}$, M. Konacki ${ }^{a}$, R. R. Thompson ${ }^{b}$, G. van Belle ${ }^{b}$, M.M. Colavita ${ }^{b}$, A.F. Boden ${ }^{b}$ \\ ${ }^{a}$ Department of Geological \& Planetary Sciences, California Institute of Technology \\ 150-21, Pasadena, CA. 91125, USA. \\ ${ }^{b}$ Jet Propulsion Laboratory, California Institute of Technology, \\ 4800 Oak Grove Dr., Pasadena, CA., 91109, USA.
}

\begin{abstract}
We discuss recent work from the Palomar Testbed Interferometer (PTI), including science results and system improvements. In the past two years PTI has been used to observe a wide range of scientifically interesting sources, including binaries, Cepheids and Miras. In addition PTI has been used to observe departures from spherical symmetry in several stars.

Recent system improvements include a new low read-noise camera based on a HAWAII infrared array, routine operation in two baselines, and operation in the $\mathbf{J}$ band. Future developments include an upgrade to three-aperture combination and closure phase measurements, and double-Fourier interferometry.
\end{abstract}

Keywords: Interferometry, Instrumentation

\section{INTRODUCTION}

The Palomar Testbed Interferometer (PTI) is a long-baseline infrared interferometer located at Palomar Observatory near San Diego, California. It typically operates in the $\mathrm{H}(1.6 \mu \mathrm{m})$ and $\mathrm{K}(2.2 \mu \mathrm{m})$ bands. It has three 40 -cm diameter apertures separated by $110-\mathrm{m}$ and $80-\mathrm{m}$ baselines, giving a maximum angular resolution of $\sim 3$ milli-arcseconds. Currently PTI combines two apertures at a time, but it is possible to switch baselines fairly rapidly for improved UV coverage.

PTI was developed by the Jet Propulsion Laboratory for NASA as a testbed for interferometric techniques applicable to the Keck Interferometer. PTI has been used in the development of high-sensitivity direct-detection interferometry in the infrared with array detectors, phase-referencing, and narrow-angle astrometry.

Major development of PTI began in November 1992 with the commencement of funding from NASA under its TOPS program. The interferometer was installed at Palomar Observatory during the spring of 1995, and first fringes were obtained in July 1995. Initial astrometric measurements were made starting in 1997, achieving 100 $\mathrm{aas}$ accuracy in 1999. Phase referencing results using long integration times were also first obtained in 1999. PTI is routinely used for several observational programs covering binary stars, Cepheids, young stellar objects and evolved stars.

\section{RECENT PTI SCIENCE}

\subsection{Binary Stars}

PTI has been successfully used to determine a number of visual orbits of double-lined spectroscopic binary systems; BY Dra (Boden and Lane 2001), 12 Boo (Boden, Creech-Eakman and Queloz 2000), 64 Psc (Boden et al. 1999a), $\iota$ Peg (Boden et al. 1999b) and RS Cvn (Koresko et al. 1998).

HD 27483 is a double-lined spectroscopic binary in the Hyades open cluster that has been recently observed wit the PTI in the $\mathrm{K}$ band. It consists of two F6 V stars orbiting each other with the period of 3.06 days. Its visual magnitude is 6.1 (5.1 in the $\mathrm{K}$ band). The distance to the system is $45.9 \mathrm{pc}$ (from the Hipparcos parallax measurement). The radial velocity orbit was determined by Northcott and Wright (1952) and improved by Mayor and Mazeh (1987). According to the latter paper the orbital parameters are: period $P=3.059120(2)$ days, time of periastron $T_{0}=2444497.685$, eccentricity $e=0.0$; and the amplitudes of the radial velocity variations are $K_{1}=72.4(5) \mathrm{km} / \mathrm{s}$ and $K_{2}=73.4(5) \mathrm{km} / \mathrm{s}$. Our $V 2$ observations allow to determine the visual orbit (see Fig. 1) and

\footnotetext{
Send correspondence to B.F.L. : E-mail: ben@gps.caltech.edu
} 
derive the masses of stars. For the purpose of this determination the brightness ratio $r=1$ was assumed as $\mathrm{K}$ band data do not allow to fit for $r$ independently of the projected semi-major axis. In order to full resolve this system, additional $\mathrm{H}$ band observations will be carried out this fall. Yet, HD 27483 remains the smallest $(a \approx 1.2 m a s)$ binary successfully observed with an optical interferometer.

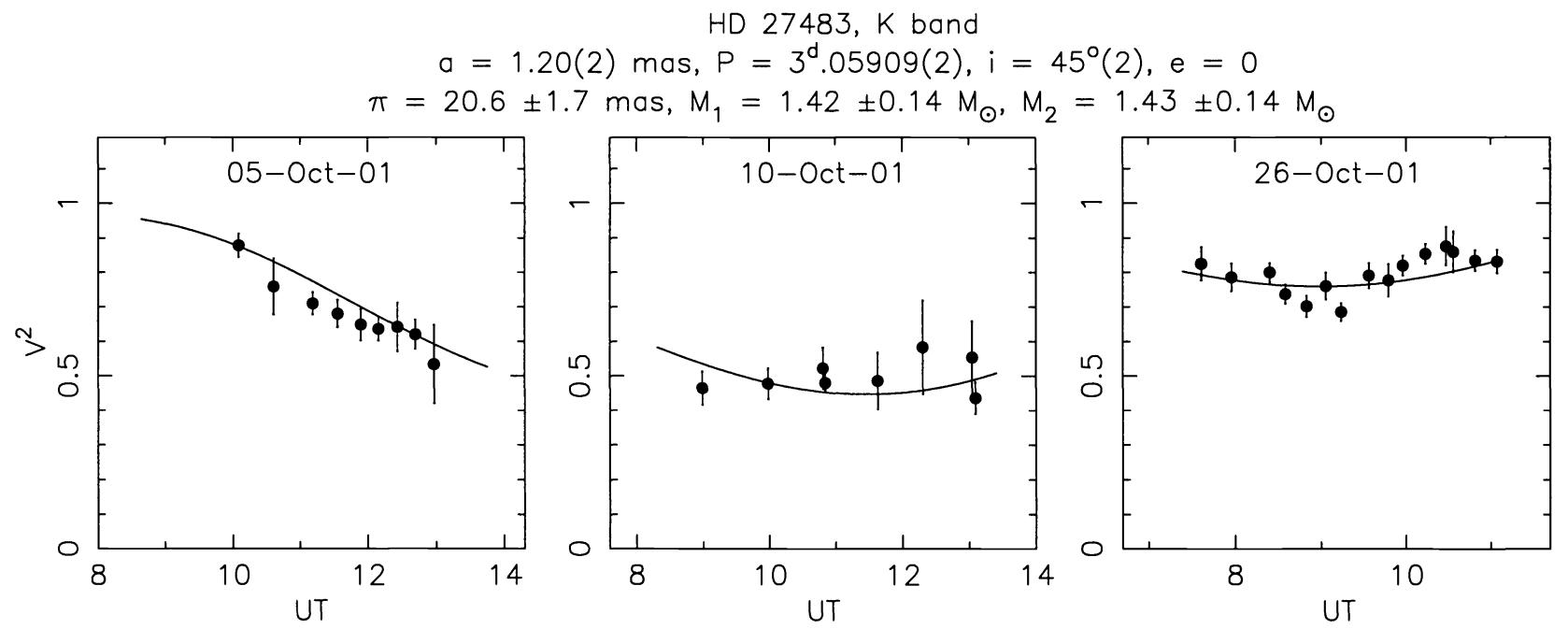

Figure 1. Calibrated $\mathrm{V}^{2}$ measurements of HD 27482 for three specific nights as a function of time (UT, in hours). The solid line represents the best fit model.

\subsection{Cepheids}

Using PTI it is possible to resolve the diameter changes undergone by nearby Cepheids during a pulsational cycle. By combining such diameter measurements with radial velocity measurements of the stellar photosphere, it is possible to determine the size of and distance to the Cepheid. Such a direct measurement is independent of photometric observations and their associated uncertainties.

PTI has been used by Lane et al. $(2000,2002)$ to observe the galactic cepheids $\eta$ Aql and $\zeta$ Gem. These observations resolved the diameter changes associated with pulsation, making it possible to determine the distance to the Cepheids at the $10 \%$ level. They also provide a measurement of the mean diameter of these Cepheids, which is useful in constructing structural models of this class of star.

\subsection{Evolved Stars}

The PTI Mira program has been following a group of about 70 M, C and S-type miras since June, 1999. Observations were generally taken every 2-3 weeks during the observing g season, with a system visibility estimated instantaneously for a given night's data based on the observations of mostly unresolved $(0.8<$ mas $)$ calibration standards, interleaved with the target observations.

Based on the wealth of data present in the PTI sample, a dissertation (Thompson 2002) and two refereed papers on some specific sources have already been published (Thompson, Creech-Eakman \& Akeson, 2002; Thompson, Creech-Eakman, \& van Belle, 2002). We briefly highlight some of the key results of these papers. The oxygen-rich Mira S Lac exhibits a minimum apparent size in the center of the K-band, typical of oxygen-rich Miras (Thompson 2002), which is not evident in the non-Mira counterpart (Fig 3). The sources of opacity, such as $\mathrm{H}_{2} \mathrm{O}$, account for the larger sizes in the 2.0 and 2.4 um channels. The carbon-rich Mira RZ Peg depicts a maximum apparent size in the 2.3 and $2.4 \mathrm{um}$ channels, owing to $\mathrm{C}_{2} \mathrm{H}_{2}, \mathrm{HCN}$ and $\mathrm{CO}$ as sources of opacity. The shape in RZ Peg is also typical of carbon-rich Miras (Thompson 2002). Multi-epoch data on S Lac (Fig 4) show a phase lag between the 2.2 and $2.4 \mathrm{um}$ apparent sizes, suggesting the source of opacity $\left(\mathrm{H}_{2} \mathrm{O}\right)$ lies above the $\tau(2.2 \mathrm{um})=1$ photosphere. No phase 

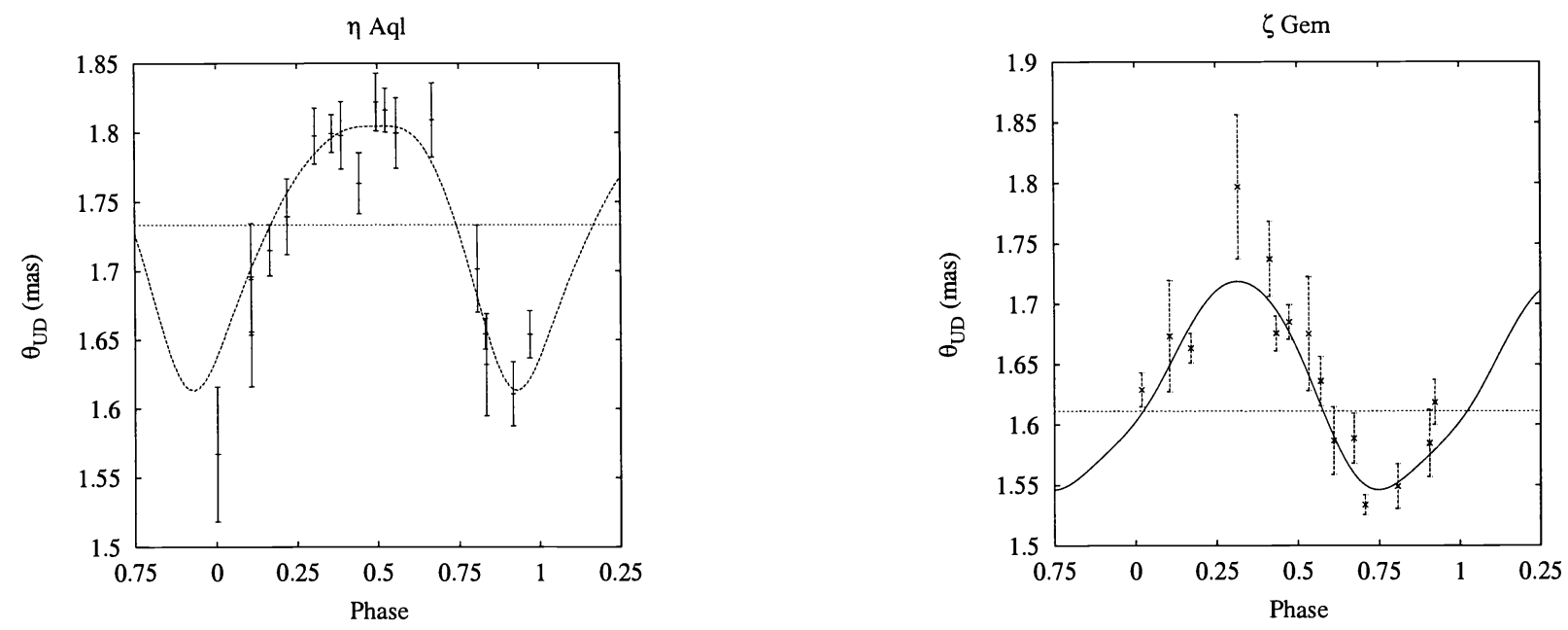

Figure 2. The angular diameters of $\eta$ Aql (left) and $\zeta$ Gem (right) as a function of pulsational phase, together with a model based on radial velocity data, but fitting for distance, mean radius and phase shift. Also shown is the result of fitting a line to all the data. Figure from Lane et al.(2002).

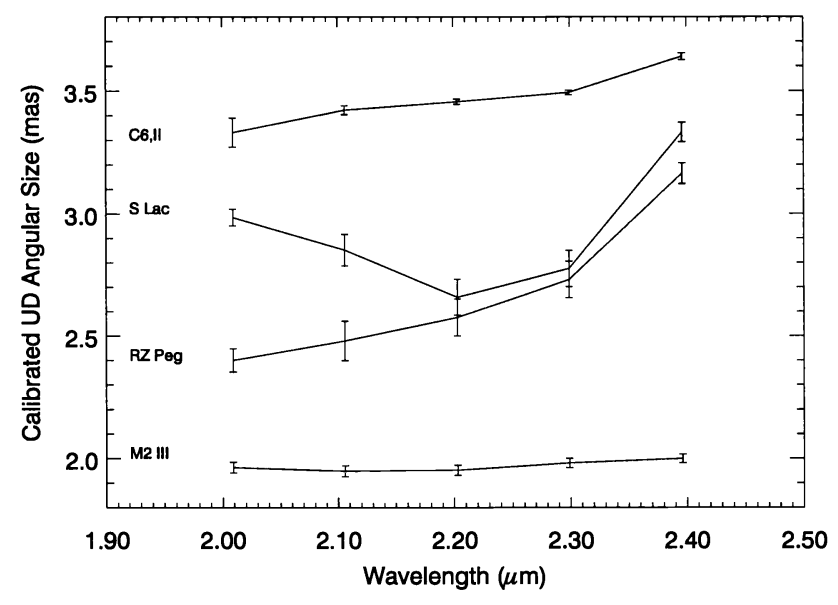

Figure 3. Calibrated uniform-disk spectral angular sizes for Miras and non-Miras. Both Mira stars exhibit greater degrees of size departures from their continuum than do the non-Mira chemical counterparts. These representative data were taken on the same night (JD 2451715). Top to Bottom: HIP 92194 (C6,II), S Lac (M4-M8e), RZ Peg (C5-6e,I), HD 193347 (M2 III). Figure from Thompson (2002).

lags are seen between the three K-band channels in RZ Peg, suggesting the sources of opacity lie at the $\tau(2.2 \mathrm{um})=1$ photosphere.

Also, series of observational programs of non-variable evolved stars has been ongoing at PTI since 1998. An early paper covering this topic in general has already been published (van Belle et al. 1999), and ensuing detailed programs are now specifically directed at giant stars, supergiant stars, and carbon stars. These observational programs are already beginning to yield information regarding the general temperature scales and atmospheric structure of these 



Figure 4. Calibrated uniform-disk spectral angular size vs. phase for RZ Peg and S Lac. The top four plots depict the red and blue band-edge sizes, the bottom two plots depict the continuum sizes overlaid with the visual magnitude data. The curves represent the best sinusoid fit to the angular size data (see Table 2). (For RZ Peg, open symbols depict observations for the first cycle, filled symbols for the second. For S Lac, open symbols depict phases $0.7-2.0$, filled symbols depict phases 2.0 - 3.8.). Figure from Thompson (2002).

objects.

\subsection{Rotationally Distorted Stars}

PTI has recently been used to measure the apparent angular diameter of several nearby, bright stars such as Vega and Altair (van Belle et al., 2001). Indications that there was an angular dependency to Altair's size were explained in terms of the star's rapid rotation rate, known from spectroscopic measures of line broadening. Contemporaneous measurements of Vega (a known slow rotator) showed no such dependency and eliminated the possibility that an instrumental effect was being observed. Modeling the star in terms of a rigid, rapidly rotating gaseous body (a Roche model) resulted in a measurement of the apparent rotation velocity of approximately $210 \mathrm{~km} / \mathrm{s}(+-10 \mathrm{~km} / \mathrm{s})$, independent of but in agreement with the spectroscopic measures. Future interferometric investigations of this nature should be able to also solve for the stellar inclination to the observer's line of sight and, as a result, the true rotation velocity, a parameter unobtainable via spectroscopic techniques. 




Figure 5. Measured angular radius as a function of projected angle on the sky for the rapid rotator Altair. Also shown is a best-fit model of the star. Figure from van Belle et al. (2001).

\section{SYSTEM IMPROVEMENTS}

\subsection{Low-Background Dewar and HAWAII Detector}

PTI has also obtained a new low-background dewar and detector. In addition, the previous design based on a warm, slitless spectrometer has been moved inside the dewar to improve the thermal background. The new detector is a Rockwell/Boeing HAWAII 1024x1024 HgCdTe array. Measurements of the detector show a read noise performance of 3-5 electrons, which should allow observations of fainter sources in the near future.

\subsection{J-band Operation}

The current beam-combination optics, although designed for operation in the $\mathrm{H}$ and $\mathrm{K}$ bands only, have sufficiently good performance in the $\mathrm{J}$ band $(1.2 \mu \mathrm{m})$ that it is possible to operate the instrument in that band. We obtained first J-band fringes in 2001, and are considering regular use of this mode for very high spatial resolution observations.

\section{FUTURE DEVELOPMENTS}

\subsection{Double-Fourier Interferometry}

The unique "dual-star" configuration of PTI allows the instrument to track two closely separated stars $(\sim 20 \operatorname{arcsec})$ and measure their relative fringe position. In addition, it is possible to track the fringe motion induced by the atmosphere using one beam combiner, and use that information to make the fringe seen by the other beam combiner appear stable. These techniques, known as phase referencing, allows a range of interesting possibilities; one idea we are beginning to explore is that of double-Fourier interferometry. In this setup the delay-stabilized starlight is fed to a Fourier transform spectrometer, making possible simultaneous high spatial and spectral resolution.

\subsection{Closure Phase}

We have begun the process of upgrading the Palomar Testbed Interferometer to simultaneous three-baseline operation. This allows true imaging and represents a novel capability for PTI. The primary purpose of this upgrade will be to further develop concepts and technology for subsequent implementation at the Keck Interferometer. It also enables science, including imaging of protoplanetary disks and close-in massive planets.

The planned beam combination scheme uses three free-space pairwise combiners and optical fibers to bring the combined beams onto the detector. The current fringe tracking approach will be used, with one broadband channel and 5-10 spectral channels. As with the single-baseline combiner, a single-mode fiber will be used to spatially filter 
the spectral channels and hence improve the quality of the measure fringe visibilities. Limiting magnitude for this system is expected to be $m_{K} \sim 4.5$, based on our experience with the previous single-baseline system.

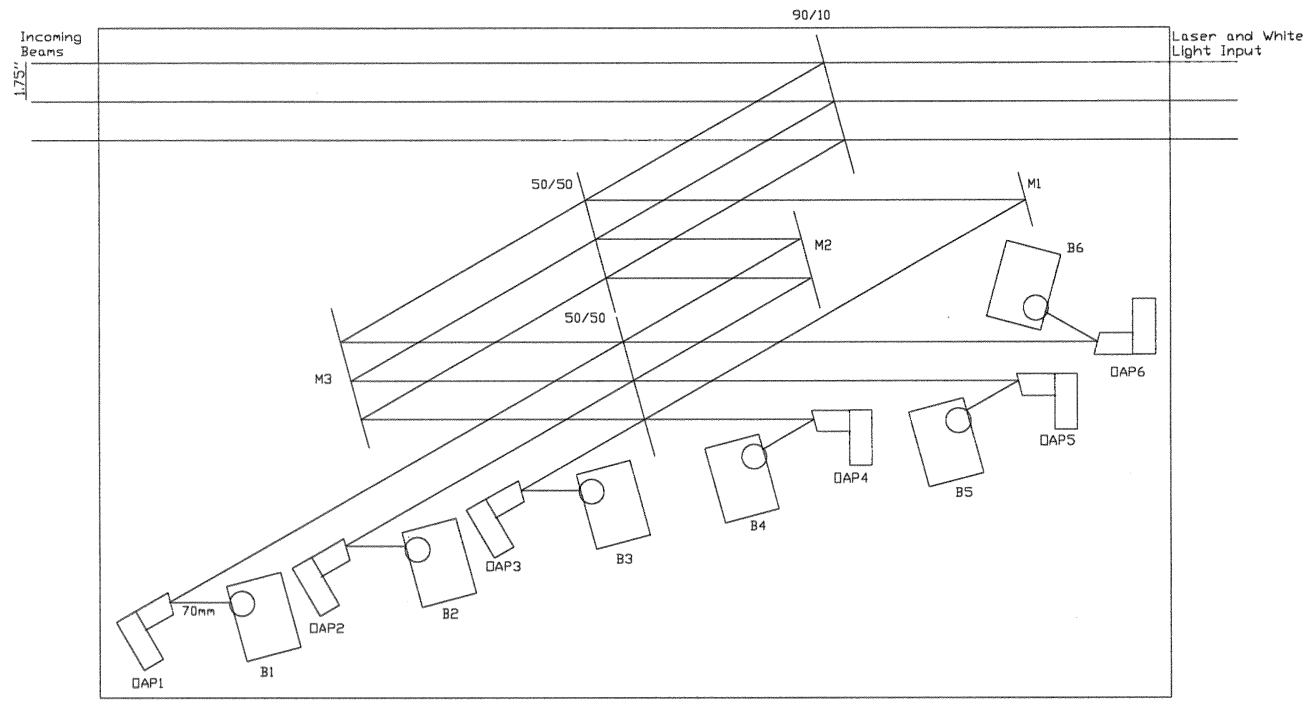

Figure 6. The planned layout for the PTI 3-way beam combiner.

\section{CONCLUSION}

In conclusion, during the past two years PTI has proved to be a very reliable and efficient instrument producing scientifically useful observations of a wide variety of interesting sources, ranging from Cepheids to binary stars. Ongoing observational programs are following Miras and Cepheids through their pulsational cycles, and we are continuing to refine the visual orbits of a number of binary stars. In addition, several new capabilities will soon be available to further extend the capabilities of this instrument.

\section{ACKNOWLEDGMENTS}

PTI was developed by the Jet Propulsion Laboratory, California Institute of Technology, under contract with the National Aeronautics and Space Administration. Funding for the development of PTI was provided by NASA under its TOPS (Toward Other Planetary Systems) and ASEPS (Astronomical Studies of Extrasolar Planetary Systems) programs, and from the JPL Director's Discretionary Fund. Ongoing funding has been provided by NASA through its Origins Program and from the JPL Directors Research and Development Fund.

\section{REFERENCES}

1. Boden, A. F. \& Lane, B. F. 2001, ApJ, 547, 1071

2. Boden, A. F., Creech-Eakman, M. J., \& Queloz, D. 2000, ApJ, 536, 880

3. Boden, A. F. et al. 1999a, ApJ, 527, 360

4. Boden, A. F. et al. 1999b, ApJ, 515, 356

5. M. Colavita et al., ApJ, 510, pp. 505-521, 1999.

6. Koresko, C. D. et al. 1998, ApJL, 509, L45

7. Lane, B. F., Kuchner, M. J., Boden, A. F., Creech-Eakman, M., Kulkarni, R. R., 2000, Nature, 407, $485-487$. 
8. Lane, B. F., Creech-Eakman, M., Nordgren, T.E., ApJ, 573, pp 330-337, 2002.

9. Northcott, R. J. \& Wright, K. O. 1952, JRASC, 46, 11

10. Mayor, M. \& Mazeh, T. 1987, A\&A, 171, 157

11. Thompson, R. R., 2002, PhD Thesis, Univ. of Wyoming.

12. Thompson, R. R., Creech-Eakman, M. J. \& Akeson, R. L., 2002, ApJ, 570, 373.

13. Thompson, R. R., Creech-Eakman, M. J. \& van Belle, G. T., 2002, ApJ, accepted.

14. van Belle, G. T., Ciardi, D. R., Thompson, R. R., Akeson, R. L., Lada, E. A., 2001, ApJ, 559, 1155-1164.

15. van Belle, G.T., et al., 1999, AJ, 117, 521. 